\title{
A Nonlinear Beam Equation Arising in the Theory of Elastic Bodies
}

\author{
K. Doppel, W. Herfort and K. Pflüger
}

\begin{abstract}
We study the global solvability of a nonlinear Cauchy problem, which arises in the theory of oscillations in elastic bodies. We show that the linearized problem defines a contraction semigroup, which is then used to transform the Cauchy problem into an integral equation. Finally, it is shown that the corresponding integral operator has a unique fixed point, which gives rise to a global solution of the original nonlinear problem.
\end{abstract}

Keywords: Nonlinear beam equations, global solvability, oscillations in elastic bodies, fixed point methods

AMS subject classiflcation: Primary 35 G 25, secondary 35 K 55, 35 K 45, 47 H 20, 73 C 50

\section{Introduction}

Beam equations as a tool to describe nonlinear vibrations of continuous systems have a long history. The mathematical modelling of such continuous systems leads to equations of the type

$$
\partial_{t}^{2} w+\partial_{\xi}^{4} w+\alpha \partial_{t} \partial_{\xi}^{4} w-\beta\left(\partial_{\xi} w\right) \partial_{\xi}^{2} w-F=0,
$$

where $\alpha \partial_{t} \partial_{\xi}^{4} w$ is an internal damping term, the nonlinear term $\beta\left(\partial_{\xi} w\right) \partial_{\xi}^{2} w$ is related to stiffness properties of the body, and the external force $F$ describes aerodynamic damping or excitation. For a general discussion of continuous systems and their mathematical modelling we refer to Nayfeh and Mook [8].

Since exact solutions are not available in general, there is a bulk of literature dealing with these equations in order to improve this situation. It is of particular importance to study the influence of the external force $F$ on the solutions of this equation. In the paper of Holmes and Marsden [6] this external force depends - linearly - on the time derivative of the solution, whereas experiments done by Blevins [2] have shown that, already in very simple configurations of the body, nonlinear terms in the time derivative may appear in $F$. In particular, he has shown that $F$ has to be a power series of the form

$$
F=a_{1} \partial_{t} w+a_{3}\left(\partial_{t} w\right)^{3}+\ldots
$$

K. Doppel: FB Math. Freie Univ., Arnimallee 3, D - 14195 Berlin

K. Pflüger: FB Math. Freie Univ., Arnimallee 3, D - 14195 Berlin

W. Herfort: Inst. Num. Ang. Math., Techn. Univ., Wiedner Hauptstr. 8-10, A - 1040 Wien

ISSN 0232-2064 / $\$ 2.50$ (C) Heldermann Verlag Berlin 
where the coefficient $a_{3}$ should be non-zero. For this reason we shall consider the following equation as a model for nonlinear oscillations of elastic bodies under external forces:

$$
\begin{aligned}
\partial_{t}^{2} w & +\partial_{\xi}^{4} w+\gamma_{1} \partial_{t} \partial_{\xi}^{4} w-\gamma_{2} \partial_{\xi}^{2} w \\
& -\gamma_{3}\left(\int_{0}^{1}\left(\partial_{\xi} w\right)^{2} d \xi\right) \partial_{\xi}^{2} w-\gamma_{4} \partial_{t} w-\gamma_{5}\left(\partial_{t} w\right)^{3}=0
\end{aligned}
$$

where $(\xi, t) \in I \times\{t \geq 0\}, I=(0,1)$, and $\gamma_{1}, \ldots, \gamma_{5}$ are real parameters, $\gamma_{1} \geq 0$. We impose initial conditions $w(\xi, 0)=w_{0}(\xi), \partial_{t} w(\xi, 0)=w_{1}(\xi)$ and, following the suggestions of engineers, we consider the boundary conditions

$$
w(0, t)=w(1, t)=\partial_{\xi}^{2} w(0, t)=\partial_{\xi}^{2} w(1, t)=0,
$$

which describe the so-called simply supported ends. We remark that boundary conditions which model clamped ends, i.e. $w(0, t)=w(1, t)=\partial_{\xi} w(0, t)=\partial_{\xi} w(1, t)=0$ lead to the Sobolev space $H_{0}^{2}(I)$ as a natural function space for this problem, while the natural boundary conditions for equation (1.1) in the space $H^{2}(I) \cap H_{0}^{1}(I)$ are

$$
w(0, t)=w(1, t)=\partial_{\xi}^{2}\left(w+\gamma_{1} \partial_{t} w\right)(0, t)=\partial_{\xi}^{2}\left(w+\gamma_{1} \partial_{t} w\right)(0, t)=0
$$

These boundary conditions were considered in the paper of Holmes and Marsden [6]. However, as already mentioned above, for applications in the field of engineering, it seems to be more reasonable to use conditions (1.2), which were suggested, for example, by Herfort and Troger in [7]. These boundary conditions lead to additional difficulties, since one cannot use the classical Sobolev space $H^{2}$, although this space would be reasonable for equations of fourth order. Instead of this one has to introduce a suitable subspace of $\mathrm{H}^{3}$ which reflects the boundary conditions (1.2) of simply supported ends.

Setting $u=w$ and $v=\partial_{t} w$ we may transform equation (1.1) into the system

$$
\left.\begin{array}{l}
\partial_{t} u=v \\
\partial_{t} v=-\partial_{\xi}^{4} u-\gamma_{1} \partial_{\xi}^{4} v+\gamma_{2} \partial_{\xi}^{2} u+\gamma_{3}\left(\int_{0}^{1}\left(\partial_{\xi} u\right)^{2} d \xi\right) \partial_{\xi}^{2} u+\gamma_{4} v+\gamma_{5} v^{3}
\end{array}\right\}
$$

where we require the boundary conditions

$$
u(0, t)=u(1, t)=v(0, t)=v(1, t)=\partial_{\xi}^{2} u(0, t)=\partial_{\xi}^{2} u(1, t)=0
$$

and initial conditions

$$
u(\xi, 0)=w_{0}(\xi) \quad \text { and } \quad v(\xi, 0)=w_{1}(\xi)
$$

Our main result is that system (1.3) under the boundary and initial conditions (1.4) resp. (1.5) is uniquely solvable and the solution is continuous and differentiable almost everywhere with respect to $t$ (Theorem 6.1).

We organize the paper as follows:

In Section 2 we introduce a Sobolev space which contains the boundary conditions (1.4). Then we reformulate the initial-boundary value problem (1.3) - (1.5) as a Cauchy problem in this space. Section 3 is devoted to the solution of the corresponding linear problem and in Section 4 we prove some estimates for the nonlinear part. In Section 5 we prove a fixed point theorem which is used in Section 6 to obtain a unique local solution of the nonlinear problem. Using uniform boundedness results, which are proved in the Appendix, we finally show that this solution is global. In a forthcoming paper we shall study the qualitative behaviour of the solution. 


\section{Sobolev spaces and the Cauchy problem}

We define

$$
H_{b}^{3}(I)=\left\{\varphi \in H^{3}(I) \mid \varphi(0)=\varphi(1)=\partial_{\xi}^{2} \varphi(0)=\partial_{\xi}^{2} \varphi(1)=0\right\}
$$

with the scalar product $\left(\varphi_{1}, \varphi_{2}\right)_{H_{b}^{3}(I)}=\left(\partial_{\xi}^{3} \varphi_{1}, \partial_{\xi}^{3} \varphi_{2}\right)_{0}$, where $(\cdot, \cdot)_{0}$ denotes the usual scalar product in $L^{2}(I)$.

Lemma 2.1. The norm induced by $(\cdot, \cdot)_{H_{b}^{3}(I)}$ on $H_{b}^{3}(I)$ is equivalent to the usual $H^{3}$-norm. In particular the inequalities

$$
\|\varphi\|_{H^{3}} \leq 2\left\|\partial_{\xi}^{3} \varphi\right\|_{0} \leq 2\|\varphi\|_{H^{3}}
$$

hold for every $\varphi \in H_{b}^{3}(I)$. get

Proof. Let $\varphi \in H_{b}^{3}(I)$. Since $\partial_{\xi}^{2} \varphi \in H_{0}^{1}(I)$ satisfies the Poincaré inequality, we

$$
\left\|\partial_{\xi}^{2} \varphi\right\|_{0} \leq\left\|\partial_{\xi}^{3} \varphi\right\|_{0} \text {. }
$$

By the Sobolev embedding theorems, $\varphi$ is continuously differentiable, and since $\varphi(0)=$ $\varphi(1)=0$, there exists $\xi_{0} \in(0,1)$ such that $\partial_{\xi} \varphi\left(\xi_{0}\right)=0$. Therefore we get $\partial_{\xi} \varphi(\xi)=$ $\int_{\xi_{0}}^{\xi} \partial_{\xi}^{2} \varphi(y) d y$ and from the Cauchy-Schwarz inequality $\left|\partial_{\xi} \varphi(\xi)\right| \leq \int_{0}^{1}\left|\partial_{\xi}^{2} \varphi(y)\right| d y \leq$ $\left\|\partial_{\xi}^{2} \varphi\right\|_{0}$, and by integration we get $\left\|\partial_{\xi} \varphi\right\|_{0} \leq\left\|\partial_{\xi}^{2} \varphi\right\|_{0}$. The usual Poincare inequality for functions $\varphi \in H_{0}^{1}(I)$ and (2.2) gives

$$
\|\varphi\|_{0} \leq\left\|\partial_{\xi} \varphi\right\|_{0} \leq\left\|\partial_{\xi}^{2} \varphi\right\|_{0} \leq\left\|\partial_{\xi}^{3} \varphi\right\|_{0}
$$

from which the inequality (2.1) is easily established

A simple consequence of Lemma 2.1 is that

$$
X=H_{b}^{3}(I) \times H_{0}^{1}(I)
$$

is a Hilbert space with respect to the scalar product

$$
\left(\left(\begin{array}{l}
u_{1} \\
v_{1}
\end{array}\right),\left(\begin{array}{l}
u_{2} \\
v_{2}
\end{array}\right)\right)_{X}=\left(\partial_{\xi}^{3} u_{1}, \partial_{\xi}^{3} u_{2}\right)_{0}+\left(\partial_{\xi} v_{1}, \partial_{\xi} v_{2}\right)_{0}
$$

Next we introduce some operators which we need in the following considerations.

Definition 2.1. Let

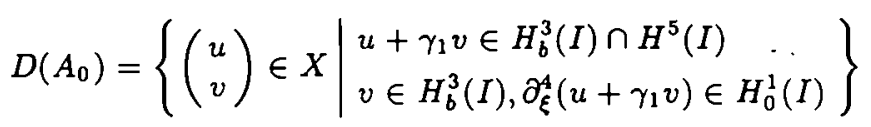


and define the operators $A_{0}: D\left(A_{0}\right) \rightarrow X, A_{1}: X \rightarrow X$ and $B: X \rightarrow X$ by

$$
\begin{aligned}
& A_{0}\left(\begin{array}{l}
u \\
v
\end{array}\right)=\left(\begin{array}{cc}
0 & 1 \\
-\partial_{\xi}^{4} & -\gamma_{1} \partial_{\xi}^{4}
\end{array}\right)\left(\begin{array}{l}
u \\
v
\end{array}\right)=\left(\begin{array}{c}
v \\
-\partial_{\xi}^{4}\left(u+\gamma_{1} v\right)
\end{array}\right) \\
& A_{1}\left(\begin{array}{l}
u \\
v
\end{array}\right)=\left(\begin{array}{cc}
0 & 0 \\
\gamma_{2} \partial_{\xi}^{2} & \gamma_{4}
\end{array}\right)\left(\begin{array}{c}
u \\
v
\end{array}\right)=\left(\begin{array}{c}
0 \\
\gamma_{2} \partial_{\xi}^{2} u+\gamma_{4} v
\end{array}\right) \\
& B\left(\begin{array}{l}
u \\
v
\end{array}\right)=\left(\begin{array}{c}
0 \\
\gamma_{3}\left\|\partial_{\xi} u\right\|_{0}^{2} \partial_{\xi}^{2} u+\gamma_{5} v^{3}
\end{array}\right),
\end{aligned}
$$

respectively, and set $G=A_{1}+B$.

Observe that for $u \in H_{b}^{3}(I)$ one has $\left\|\partial_{\xi} u\right\|_{0}^{2} \partial_{\xi}^{2} u \in H_{0}^{1}(I)$, and since $H_{0}^{1}(I)$ is a Banach algebra (cf. [1: p. 115]), $v^{3} \in H_{0}^{1}(I)$. Therefore, $B$ is well defined on $X$. Since $\partial_{\xi}^{2} u \in H_{0}^{1}(I)$, we obtain that $A_{1}$ is a bounded linear operator on $X$.

Using Definition 2.1 we reformulate problem (1.3) - (1.5) as the following

Problem A (Nonlinear Cauchy Problem). For given $\left(\begin{array}{l}u_{0} \\ v_{0}\end{array}\right) \in D\left(A_{0}\right)$ find a solution $\left(\begin{array}{l}u \\ v\end{array}\right) \in C([0, \infty), X) \cap C^{1}((0, \infty), X)$ of the problem

$$
\begin{aligned}
& \frac{d}{d t}\left(\begin{array}{l}
u \\
v
\end{array}\right)=A_{0}\left(\begin{array}{l}
u \\
v
\end{array}\right)+G\left(\begin{array}{l}
u \\
v
\end{array}\right) \\
& \left(\begin{array}{l}
u \\
v
\end{array}\right)(0)=\left(\begin{array}{l}
u_{0} \\
v_{0}
\end{array}\right) .
\end{aligned}
$$

\section{Solution of the linear problem}

The linear Cauchy problem associated to problem (2.5) - (2.6) is the following

Problem B (Linear Cauchy Problem). For given $\left(\begin{array}{l}u_{0} \\ v_{0}\end{array}\right) \in D\left(A_{0}\right)$ find a solution $\left(\begin{array}{l}u \\ v\end{array}\right) \in C((0, \infty), X) \cap C^{1}((0, \infty), X)$ of the problem

$$
\begin{aligned}
& \frac{d}{d t}\left(\begin{array}{l}
u \\
v
\end{array}\right)=A_{0}\left(\begin{array}{l}
u \\
v
\end{array}\right) \\
& \left(\begin{array}{l}
u \\
v
\end{array}\right)(0)=\left(\begin{array}{l}
u_{0} \\
v_{0}
\end{array}\right) .
\end{aligned}
$$

To solve the linear problem, we show that $A_{0}$ is the generator of a strongly continuous contraction semigroup on $X$. For this we need the following results.

Lemma 3.1. The set $D\left(A_{0}\right)$ is dense in $X$.

Proof. Fix $\left(\begin{array}{l}u \\ v\end{array}\right) \in X$. Since $v \in H_{0}^{1}(I)$ and $C_{0}^{\infty}(I)$ is dense in $H_{0}^{1}(I)$, it is sufficient to show that there exists a function $\phi \in C^{\infty}(I)$ that approximates $u$ (in the sense that $\left\|\partial_{\xi}^{3} \phi-\partial_{\xi}^{3} u\right\|_{0}$ is small) and satisfies the boundary conditions $\phi(0)=\phi(1)=$ 
$\partial_{\xi}^{2} \phi(0)=\partial_{\xi}^{2} \phi(1)=\partial_{\xi}^{4} \phi(0)=\partial_{\xi}^{4} \phi(1)=0$. Since $\partial_{\xi}^{3} u$ is in $L^{2}(I)$, we can find to any $\varepsilon>0$ a function $\varphi \in C_{0}^{\infty}(I)$ such that $\left\|\varphi-\partial_{\xi}^{3} u\right\|_{0}<\varepsilon$. We define

$$
\tilde{\varphi}(\xi)=\int_{0}^{\xi} \varphi(y) d y \quad(\xi \in I)
$$

Obviously, $\widetilde{\varphi}(0)=0$, and since $\partial_{\xi}^{3} u$ is in $L^{1}(I)$, it satisfies $\partial_{\xi}^{2} u(1)-\partial_{\xi}^{2} u(0)=\int_{0}^{1} \partial_{\xi}^{3} u(\xi) d \xi$ (cf. [4: p. 235]). The left-hand side of this equation is zero (this follows from $u \in H_{b}^{3}(I)$ ) and the right-hand side is approximately equal to $\int_{0}^{1} \varphi(\xi) d \xi$ (since $\varphi$ approximates $\partial_{\xi}^{3} u$ in the $L^{1}$-norm, too). After a suitable change of $\varphi$ we may assume that $\int_{0}^{1} \varphi(\xi) d \xi=0$, which implies $\widetilde{\varphi}(1)=0$. Next we consider

$$
\hat{\varphi}(\xi)=\int_{0}^{\xi} \tilde{\varphi}(y) d y+C \quad \text { and } \quad \phi(\xi)=\int_{0}^{\xi} \hat{\varphi}(y) d y \quad(\xi \in I)
$$

where $C$ is an arbitrary constant. Again we have $\phi(0)=0$ and

$$
\phi(1)=\int_{0}^{1} \widehat{\varphi}(\xi) d \xi=\int_{0}^{1}\left(\int_{0}^{\xi} \tilde{\varphi}(y) d y+C\right) d \xi=\int_{0}^{1} \int_{0}^{\xi} \widetilde{\varphi}(y) d y d \xi+C
$$

Choosing $C=-\int_{0}^{1} \int_{0}^{\xi} \tilde{\varphi}(y) d y d \xi$, we get $\phi(1)=0$. So we have proved $\partial_{\xi}^{2} \phi(0)=\tilde{\varphi}(0)=$ $0=\widetilde{\varphi}(1)=\partial_{\xi}^{2} \phi(1)$ and $\partial_{\xi}^{3} \phi=\varphi \in C_{0}^{\infty}(I)$. Therefore, $\phi$ is the desired function and we obtain the density of $D\left(A_{0}\right)$ in $X$

Lemma 3.2. The operator $-A_{0}$ is accretive, i.e. $\left(x, A_{0} x\right)_{X} \leq 0$ for every $x \in$ $D\left(A_{0}\right)$.

Proof. For $x=\left(\begin{array}{l}u \\ v\end{array}\right) \in D\left(A_{0}\right)$ we get by partial integration

$$
\begin{aligned}
\left(x, A_{0} x\right)_{X} & =\left(\left(\begin{array}{l}
u \\
v
\end{array}\right),\left(\begin{array}{c}
v \\
-\partial_{\xi}^{4}\left(u+\gamma_{1} v\right)
\end{array}\right)\right)_{X} \\
& =\left(\partial_{\xi}^{3} u, \partial_{\xi}^{3} v\right)_{0}+\left(\partial_{\xi} v,-\partial_{\xi}^{5}\left(u+\gamma_{1} v\right)\right)_{0} \\
& =\left(\partial_{\xi}^{3} u, \partial_{\xi}^{3} v\right)_{0}-\left(\partial_{\xi}^{3} v, \partial_{\xi}^{3}\left(u+\gamma_{1} v\right)\right)_{0} \\
& =-\gamma_{1}\left(\partial_{\xi}^{3} v, \partial_{\xi}^{3} v\right)_{0} \\
& \leq 0
\end{aligned}
$$

since $\gamma_{1} \geq 0$

Lemma 3.3. For any positive $\lambda$ the operator $\lambda I-A_{0}$ is surjective. 
Proof. Given $y=\left(\begin{array}{l}\varphi \\ \eta\end{array}\right) \in X$, we have to find an $x=\left(\begin{array}{l}u \\ v\end{array}\right) \in D\left(A_{0}\right)$ such that $\left(\lambda I-A_{0}\right) x=y$, i.e. we have to solve

$$
\left(\begin{array}{c}
\lambda u-v \\
\lambda v+\partial_{\xi}^{4}\left(u+\gamma_{1} v\right)
\end{array}\right)=\left(\begin{array}{c}
\varphi \\
\eta
\end{array}\right)
$$

This is equivalent to the determination of a function $u \in H_{b}^{3}(I)$ which satisfies

$$
\lambda^{2} u+\partial_{\xi}^{4}\left(u+\lambda \gamma_{1} u-\gamma_{1} \varphi\right)=\eta+\lambda \varphi
$$

Setting $w=\left(1+\lambda \gamma_{1}\right) u-\gamma_{1} \varphi$, equation (3.3) can be written in the form

$$
\partial_{\xi}^{4} w+\frac{\lambda^{2}}{1+\lambda \gamma_{1}} w=\eta+\frac{\lambda}{1+\lambda \gamma_{1}} \varphi
$$

Since the right-hand side of this equation belongs to $H_{0}^{1}(I)$ and the homogeneous boundary value problem

$$
\left.\begin{array}{r}
\partial_{\xi}^{4} w+\frac{\lambda^{2}}{1+\lambda \gamma_{1}} w=0 \\
w(0)=w(1)=\partial_{\xi}^{2} w(0)=\partial_{\xi}^{2} w(1)=0
\end{array}\right\}
$$

has only the trivial solution, it follows that equation (3.4) has a unique solution in $H_{b}^{3}(I) \cap H^{5}(I)$. Setting

$$
u=\frac{1}{1+\lambda \gamma_{1}} w+\frac{\gamma_{1}}{1+\lambda \gamma_{1}} \varphi \quad \text { and } \quad v=\frac{\lambda}{1+\lambda \gamma_{1}} w-\frac{1}{1+\lambda \gamma_{1}} \varphi
$$

we obtain that $u+\gamma_{1} v=w$ and $\partial_{\xi}^{4}\left(u+\gamma_{1} v\right) \dot{\in} H_{0}^{1}(I)$. Therefore, $\left(\begin{array}{l}u \\ v\end{array}\right) \in D\left(A_{0}\right)$ is the desired solution

Theorem 3.1. Let the operator $A_{0}$ be as in Definition 2.1. Then there exists a strongly continuous contraction semigroup $S$ with the following properties:

(i) For $x_{0} \in X$ the map $t \mapsto x(t):=S(t) x_{0}$ belongs to $C([0, \infty), X)$.

(ii) For $x_{0} \in D\left(A_{0}\right)$ the map $t \mapsto x(t):=S(t) x_{0}$ belongs to $C^{1}((0, \infty), X)$ and is the unique solution of the Cauchy problem $\frac{d}{d t} x(t)=A_{0} x(t), x(0)=x_{0}$.

(iii) For $x_{0} \in X$ and $t \geq 0$, the inequality $\left\|S(t) x_{0}\right\|_{X} \leq\left\|x_{0}\right\|_{X}$ holds.

Proof. Since $D\left(A_{0}\right)$ is dense in $X$ (Lemma 3.1), $-A_{0}$ is accretive (Lemma 3.2) and $\lambda I-A_{0}$ is surjective for positive $\lambda$.(Lemma 3.3 ), the assertions follow from [11: Theorem IV, 4.C]

Remark that in fact it is not necessary to prove that the domain of the operator $A_{0}$ is dense in $X$ (see [11: Problem IV, 4.2]).

For later use we state the following 
Lemma 3.4. If $x \in C([0, \infty) ; X)$, then the mapping $t \mapsto S(t) x(t)$ belongs to $C([0, \infty) ; X)$.

Proof. Let $t \in[0 ; \infty)$ and $h \in \mathbb{R}$ such that $t+h \in[0, \infty)$. We obtain

$$
\begin{aligned}
\| S(t & +h) x(t+h)-S(t) x(t) \|_{X} \\
& \leq\|S(t+h)(x(t+h)-x(t))\|_{X}+\|(S(t+h)-S(t)) x(t)\|_{X} \\
& \leq\|x(t+h)-x(t)\|_{X}+\|(S(t+h)-S(t)) x(t)\|_{\dot{X}}
\end{aligned}
$$

Since $x$ is continuous and $S$ is strongly continuous, we can find to any $\varepsilon>0$ a $\delta>0$, such that

$$
\|x(t+h)-x(t)\|_{X}<\frac{\varepsilon}{2} \quad \text { and } \quad\|(S(t+h)-S(t)) x(t)\|_{X}<\frac{\varepsilon}{2}
$$

hold simultaneously for $|h|<\delta$. This shows that $t \rightarrow S(t) x(t)$ is continuous

\section{Estimates for the operator $G$}

For the solution of the nonlinear problem, we shall need some estimates for the nonlinear operator $G$.

Lemma 4.1. The operator $G: X \rightarrow X$ from Definition 2.1 is bounded, i.e. for every bounded subset $E \subset X$ there is a constant $C_{R}$ only depending on $E$ and $\gamma_{2}, \ldots, \gamma_{5}$, such that $\|G(x)\|_{X} \leq C_{R}$ for every $x \in E$.

Proof. We choose $R>0$ such that $\left|\gamma_{i}\right| \leq R \quad(i=2, \ldots, 5)$ and $\|x\|_{X} \leq R$ for $x \in E$. We set $x=\left(\begin{array}{l}u \\ v\end{array}\right)$. For the linear operator $A_{1}$ we get

$$
\begin{aligned}
\left\|A_{1} x\right\|_{X} & =\left\|\left(\begin{array}{c}
0 \\
\gamma_{2} \partial_{\xi}^{2} u+\gamma_{4} v
\end{array}\right)\right\|_{X} \\
& =\left\|\gamma_{2} \partial_{\xi}^{3} u+\dot{\gamma}_{4} \partial_{\xi} v\right\|_{0} \leq R\left(\left\|\partial_{\xi}^{3} u\right\|_{0}+\left\|\partial_{\xi} v\right\|_{0}\right) \leq 2 R^{2} .
\end{aligned}
$$

To estimate the nonlinear operator $B$, we remark that $v \in H_{0}^{1}(I)$ is continuous and satisfies $v(\xi)=\int_{0}^{\xi} \partial_{\xi} v(y) d y$. Using the Cauchy-Schwarz inequality; we obtain

$$
|v(\xi)| \leq\left\|\partial_{\xi} v\right\|_{0} . \quad \text { for } \xi \in I
$$

We claim that

$$
\left\|\partial_{\xi}\left(v^{3}\right)\right\|_{0}=3\left\{\int_{0}^{1}|v(\xi)|^{4}\left|\partial_{\xi} v(\xi)\right|^{2} d \xi\right\}^{\frac{1}{2}} \leq 3\left\|\partial_{\xi} v\right\|_{0}^{2}\left\{\int_{0}^{1}\left|\partial_{\xi} v(\xi)\right|^{2} d \xi\right\}^{\frac{1}{2}}=3\left\|\partial_{\xi} v\right\|_{0}^{3}
$$

and get

$$
\begin{aligned}
\|B(x)\|_{X} & =\left\|\left(\begin{array}{c}
0 \\
\gamma_{3}\left\|\partial_{\xi} u\right\|_{0}^{2} \partial_{\xi}^{2} u+\gamma_{5} v^{3}
\end{array}\right)\right\|_{X} \\
& =\left\|\gamma_{3}\right\| \partial_{\xi} u\left\|_{0}^{2} \partial_{\xi}^{3} u+\gamma_{5} \partial_{\xi}\left(v^{3}\right)\right\|_{0} \leq R\left(\left\|\partial_{\xi}^{3} u\right\|_{0}^{3}+3\left\|\partial_{\xi} v\right\|_{0}^{3}\right) \leq 4 R^{4}
\end{aligned}
$$

where we have used (2.3). This shows that $C_{R}=2 R^{2}+4 R^{4}$ is the desired constant 
Lemma 4.2. For every bounded subset $E \subset X$ there is a constant $D_{R}$ only depending on $E$ and $\gamma_{2}, \ldots, \gamma_{5}$, such that $\left\|G\left(x_{1}\right)-G\left(x_{2}\right)\right\|_{X} \leq D_{R}\left\|x_{1}-x_{2}\right\|_{X}$ for $x_{1}, x_{2} \in E$.

Proof. Choose $R>0$ such that $\left|\gamma_{i}\right| \leq R \quad(i=2, \ldots, 5)$ and $\|x\|_{X} \leq R$ for $x \in E$. Then we get (setting $x_{i}=\left(\begin{array}{l}u_{i} \\ v_{i}\end{array}\right)$ for $i=1,2$ )

$$
\begin{aligned}
\| G\left(x_{1}\right) & -G\left(x_{2}\right) \|_{X} \\
= & \| \gamma_{2}\left(\partial_{\xi}^{3} u_{1}-\partial_{\xi}^{3} u_{2}\right)+\gamma_{4}\left(\partial_{\xi} v_{1}-\partial_{\xi} v_{2}\right) \\
& +\gamma_{3}\left(\left\|\partial_{\xi} u_{1}\right\|_{0}^{2} \partial_{\xi}^{3} u_{1}-\left\|\partial_{\xi} u_{2}\right\|_{0}^{2} \partial_{\xi}^{3} u_{2}\right)+\gamma_{5}\left(\partial_{\xi} v_{1}^{3}-\partial_{\xi} v_{2}^{3}\right) \|_{0} \\
\leq & \left|\gamma_{2}\right|\left\|\partial_{\xi}^{3}\left(u_{1}-u_{2}\right)\right\|_{0}+\left|\gamma_{4}\right|\left\|\partial_{\xi}\left(v_{1}-v_{2}\right)\right\|_{0} \\
& +\left|\gamma_{3}\right|\left\{\left\|\partial_{\xi} u_{1}\right\|_{0}^{2}\left\|\partial_{\xi}^{3} u_{1}-\partial_{\xi}^{3} u_{2}\right\|_{0}+\left\|\partial_{\xi}^{3} u_{2}\right\|_{0}\left|\left\|\partial_{\xi} u_{1}\right\|_{0}^{2}-\left\|\partial_{\xi} u_{2}\right\|_{0}^{2}\right|\right\} \\
& +\left|\gamma_{5}\right|\left\|\partial_{\xi}\left\{\left(v_{1}-v_{2}\right)\left(v_{1}^{2}+v_{1} v_{2}+v_{2}^{2}\right)\right\}\right\|_{0} .
\end{aligned}
$$

From (4.1) we get the estimate

$$
\begin{aligned}
\left\|\partial_{\xi}\left(v_{1}^{2}+v_{1} v_{2}+v_{2}^{2}\right)\right\|_{0} & \leq 2\left\|v_{1} \partial_{\xi} v_{1}\right\|_{0}+\left\|v_{1} \partial_{\xi} v_{2}\right\|_{0}+\left\|v_{2} \partial_{\xi} v_{1}\right\|_{0}+2\left\|v_{2} \partial_{\xi} v_{2}\right\|_{0} \\
& \leq 2\left\|\partial_{\xi} v_{1}\right\|_{0}^{2}+\left\|\partial_{\xi} v_{2}\right\|_{0}^{2}+\left\|\partial_{\xi} v_{1}\right\|_{0}^{2}+2\left\|\partial_{\xi} v_{2}\right\|_{0}^{2}
\end{aligned}
$$

From $\left\|v_{i}\right\|_{0} \leq\left\|\partial_{\xi} v_{i}\right\|_{0} \leq\left\|x_{i}\right\|_{X} \leq R$ and $\left\|\partial_{\xi} u_{i}\right\|_{0} \leq\left\|\partial_{\xi}^{3} u_{i}\right\|_{0} \leq\left\|x_{i}\right\|_{X} \leq R$ we finally obtain

$$
\begin{aligned}
\left\|G\left(x_{1}\right)-G\left(x_{2}\right)\right\|_{X} \leq & 2 R\left\|x_{1}-x_{2}\right\|_{X}+R^{3}\left\|x_{1}-x_{2}\right\|_{X} \\
& +2 R^{3}\left\|x_{1}-x_{2}\right\|_{X}+3 R^{3}\left\|x_{1}-x_{2}\right\|_{X}+6 R^{3}\left\|x_{1}-x_{2}\right\|_{X} .
\end{aligned}
$$

Therefore $D_{R}=2 R+12 R^{3}$ is the desired constant

\section{A fixed point theorem}

Let $Y$ be a (real) Banach space whith norm $\|\cdot\|_{Y}$. For fixed real numbers $0 \leq t_{0}<t_{1}$ consider the space $C\left(\left[t_{0}, t_{1}\right], Y\right)$ of continuous Banach-valued functions over the (closed) interval $\left[t_{0}, t_{1}\right]$. Define for a fixed $y_{0} \in Y$ the $R$-ball of functions from $C\left(\left[t_{0}, t_{1}\right], Y\right)$ by

$$
B\left(y_{0}, R\right)=\left\{\phi \in \dot{C}\left(\left[t_{0}, t_{1}\right], Y\right) \mid\left\|\phi(t)-y_{0}\right\|_{Y} \leq R \quad\left(t \in\left[t_{0}, t_{1}\right]\right)\right\}
$$

Then we can prove the following

Theorem 5.1. Fix $y_{0} \in Y$ and assume that the function

$$
F: C\left(\left[t_{0}, t_{1}\right], Y\right) \rightarrow C\left(\left[t_{0}, t_{1}\right], Y\right)
$$

has the following properties: 
(i) There exists $R>0$ such that $\left\|F(\phi)(t)-y_{0}\right\|_{Y} \leq R$ for all $\phi \in B\left(y_{0}, R\right)$ and all $t \in\left[t_{0}, t_{1}\right]$.

(ii) There exists $K_{R}>0$ such that $\|F(\phi)(t)-F(\psi)(t)\|_{Y} \leq K_{R} \int_{t_{0}}^{t}\|\phi(s)-\psi(s)\|_{Y} d s$ for all $\phi, \psi \in B\left(y_{0}, R\right)$ and all $t \in\left[t_{0}, t_{1}\right]$.

Then $F$ has a unique fixed point $\phi_{\infty} \in B\left(y_{0}, R\right)$. Furthermore, the sequence $\left\{\phi_{n}\right\}_{n \geq 0}$ defined by $\phi_{0}(t)=y_{0}$ and $\phi_{n+1}(t)=F\left(\phi_{n}\right)(t) \quad(n \geq 0)$ converges uniformly with respect to $t$ to the fixed point $\phi_{\infty}$.

This fixed point theorem is a consequence of a slightly more general result of Foias, Gussi and Poenaru [5]. For the reader's convenience we shall give here an independent, more elementary proof.

Proof of Theorem 5.1. 1. We show by induction that for any integer $j \geq 0$ the estimate

$$
\left\|\phi_{j+1}(t)-\phi_{j}(t)\right\|_{Y} \leq R \frac{K_{R}^{j}\left(t-t_{0}\right)^{j}}{j !}
$$

is valid for all $t \in\left[t_{0}, t_{1}\right]$. From property $(i)$ we see that $(5.1)$ is certainly true for $j=0$. Assume that (5.1) has been shown for $k=0, \ldots, j$. Then from property (ii) it follows that

$$
\begin{aligned}
\left\|\phi_{j+2}(t)-\phi_{j+1}(t)\right\|_{Y} & =\left\|F\left(\phi_{j+1}\right)(t)-F\left(\phi_{j}\right)(t)\right\|_{Y} \\
& \leq K_{R} \int_{t_{0}}^{t}\left\|\phi_{j+1}(s)-\phi_{j}(s)\right\|_{Y} d s \\
& \leq R K_{R} \int_{t_{0}}^{t} \frac{K_{R}^{j}\left(s-t_{0}\right)^{j}}{j !} d s \\
& =R \frac{K_{R}^{j+1}\left(t-t_{0}\right)^{j+1}}{(j+1) !} .
\end{aligned}
$$

2. We show that $\left\{\phi_{n}\right\}_{n \geq 0}$ is a Cauchy sequence with respect to the uniform norm in $C\left(\left[t_{0}, t_{1}\right], Y\right)$ and converges to a fixed point of $F$. Therefore we estimate

$$
\begin{aligned}
\left\|\phi_{n+p}(t)-\phi_{n}(t)\right\|_{Y} & \leq \sum_{j=0}^{p-1}\left\|\phi_{n+j+1}(t)-\phi_{n+j}(t)\right\|_{Y} \\
& \leq R K_{R}^{n}\left(t-t_{0}\right)^{n} \sum_{j=0}^{p-1} \frac{K_{R}^{j}\left(t-t_{0}\right)^{j}}{(n+j) !} \\
& \leq R K_{R}^{n} \frac{\left(t_{1}-t_{0}\right)^{n}}{n !} \sum_{j=0}^{p-1} \frac{K_{R}^{j}\left(t_{1}-t_{0}\right)^{j}}{j !} \\
& \leq R \exp \left(K_{R}\left(t_{1}-t_{0}\right)\right) \frac{K_{R}^{n}\left(t_{1}-t_{0}\right)^{n}}{n !}
\end{aligned}
$$

(where we have used the inequality $(n+j) ! \geq n ! j !)$. Consequently, we get the uniform convergence of $\phi_{n}$ to an element $\phi_{\infty}$ and the a priori estimate

$$
\left\|\phi_{\infty}(t)-\phi_{n}(t)\right\|_{Y} \leq R \exp \left(K_{R}\left(t_{1}-t_{0}\right)\right) \frac{K_{R}^{n}\left(t_{1}-t_{0}\right)^{n}}{n !} \quad\left(t \in\left[t_{0}, t_{1}\right]\right) .
$$


Since $F$ is continuous with respect to the uniform norm, we can choose for each $\varepsilon>0$ an $n_{\varepsilon} \in \mathbb{N}$ such that

$$
\begin{aligned}
& \left\|F\left(\phi_{\infty}\right)(t)-\phi_{\infty}(t)\right\|_{Y} \\
& \quad \leq\left\|F\left(\phi_{\infty}\right)(t)-F\left(\phi_{n}\right)(t)\right\|_{Y}+\left\|\phi_{n+1}(t)-\phi_{\infty}(t)\right\|_{Y}<\varepsilon
\end{aligned}
$$

holds uniformly in $t$ for every $n \geq n_{\varepsilon}$. This shows that $\phi_{\infty}$ is a fixed point of $F$.

3. The fixed point is unique. Indeed, assume that $F(\phi)=\phi$ and $F(\psi)=\psi$. Successive application of property (ii) leads to

$$
\begin{aligned}
\|\phi(t)-\psi(t)\|_{Y} & =\|F(\phi)(t)-F(\psi)(t)\|_{Y} \\
& \leq K_{R} \int_{t_{0}}^{t}\left\|\phi\left(s_{1}\right)-\psi\left(s_{1}\right)\right\|_{Y} d s_{1} \\
& =K_{R} \int_{t_{0}}^{t}\left\|\phi\left(s_{1}\right)-\psi\left(s_{1}\right)\right\|_{Y} d s_{1} \\
& \vdots \\
\leq & K_{R}^{n} \int_{t_{0}}^{t} \int_{t_{0}}^{s_{1}} \cdots \int_{t_{0}}^{s_{n-1}}\left\|\phi\left(s_{n}\right)-\psi\left(s_{n}\right)\right\|_{Y} d s_{n} d s_{n-1} \cdots d s_{1} \\
\leq & K_{R}^{n} \int_{t_{0}}^{t} \int_{t_{0}}^{s_{1}} \cdots \int_{t_{0}}^{s_{n-1}}\left\|\phi\left(s_{n}\right)-y_{0}\right\|_{Y} d s_{n} d s_{n-1} \cdots d s_{1} \\
& +K_{R}^{n} \int_{t_{0}}^{s_{1}} \int_{t_{0}}^{s_{1}} \cdots \int_{t_{0}}^{s_{n-1}}\left\|y_{0}-\psi\left(s_{n}\right)\right\|_{Y} d s_{n} d s_{n-1} \cdots d s_{1} \\
& \leq 2 R K_{R}^{n} \frac{\left(t_{1}-t_{0}\right)^{n}}{n !} \vdots
\end{aligned}
$$

Since the last term tends to zero as $\dot{n}^{\rightarrow} \rightarrow \infty$, we see that $\phi(t)=\psi(t)$ for every $t \in\left[t_{0}, t_{1}\right]$ and the proof is complete

\section{Solution of the nonlinear problem}

If $x \in C\left(\left[t_{0}, t_{1}\right], X\right)$ is a solution of the Cauchy problem

$$
\left.\begin{array}{rl}
\frac{d}{d t} x(t) & =A_{0} x(t)+G(x(t)) \\
x\left(t_{0}\right) & =x_{0} \in D\left(A_{0}\right),
\end{array}\right\}
$$


then it is also a solution of the integral equation

$$
x(t)=S\left(t-t_{0}\right) x_{0}+\int_{t_{0}}^{t} S(t-s) G(x(s)) d s .
$$

A continuous solution $x$ of this integral equation is called a mild solution, and it is called a strong solution if $x$ is differentiable a.e. with $x^{\prime} \in L^{1}\left(\left(t_{0}, t_{1}\right), X\right)$. First we shall use the fixed point theorem of the last section to construct a unique (local) solution of equation (6.2). In Theorem 6.1 below we shall prove that this is in fact a strong solution which exists globally in time.

Let $X$ be the Hilbert space (2.4) and define the mapping $F: C\left(\left[t_{0}, t_{1}\right], X\right) \rightarrow$ $C\left(\left[t_{0}, t_{1}\right], X\right)$ by

$$
F(x)(t)=S\left(t-t_{0}\right) x_{0}+\int_{t_{0}}^{t} S(t-s) G(x(s)) d s
$$

where $x_{0}$ is a fixed element in $X, S$ is the sernigroup of Theorem 3.1 , and $G$ is the operator of Definition 2.1. To use the fixed point Theorem 5.1 for this function, we have to check that there exist real numbers $0 \leq t_{0}<t_{1}$ such that $F$ satisfies the conditions (i) and (ii). Therefore, we prove the following

Lemma 6.1. Fix $x_{0} \in X$ and let $F$ be defined by (6.3). Then there exist real numbers $R>0$ and $0 \leq t_{0}<t_{1}$ such that

$$
\left\|F(\phi)(t)-x_{0}\right\| x \leq R
$$

is valid for all $\phi \in B\left(x_{0}, R\right)$ and for all $t \in\left[t_{0}, t_{1}\right]$.

We give the proof of this lemma by the help of the following

Proposition 6.1. Let $x_{0} \in X$ be given and choose $R>0$ such that $R \geq\left|\gamma_{i}\right|:(i=$ $2, \ldots, 5)$ and $R \geq\left\|x_{0}\right\|_{X}$. Then for any positve $\varepsilon<R$ there exists a $\delta\left(x_{0}, \varepsilon\right)$ such that for $t_{0} \leq t \leq t_{0}+\delta\left(x_{0}, \varepsilon\right)$ the inequality

$$
\left\|F(\phi)(t)-x_{0}\right\|_{X}<\varepsilon+C_{2 R}\left(t-t_{0}\right)
$$

holds whenever $\left\|\phi(t)-x_{0}\right\|_{X} \leq R$, where $C_{2 R}$ is the constant from Lemma 4.1 corresponding to $2 R$.

Proof. Fix $t_{0} \geq 0$. Since $S$ is strongly continuous, tò $\varepsilon>0$ we find a $\delta\left(x_{0}, \varepsilon\right)>0$ such that $\left\|S\left(t-t_{0}\right) x_{0}-x_{0}\right\|_{x}<\varepsilon$ for $t_{0} \leq t \leq t_{0}+\delta\left(x_{0}, \varepsilon\right)$. If $\left\|\phi(t)-x_{0}\right\| x \leq R$, we have $\|\phi(t)\|_{X} \leq 2 R$. Therefore from Lemma 4.1 we obtain a constant $C_{2 R}$ such that $\|G(\phi)(t)\|_{X} \leq C_{2 R}$ holds if $\left\|\phi(t)-x_{0}\right\|_{X} \leq R$. Consequently we get

$$
\begin{aligned}
\left\|F(\phi)(t)-x_{0}\right\|_{x} & \leq\left\|S\left(t-t_{0}\right) x_{0}-x_{0}\right\| x+\int_{t_{0}}^{t}\|S(t-s) G(\phi(s))\| x d s \\
\because \quad \therefore \quad & \leq<\varepsilon+\int_{t_{0}}^{t}\|G(\phi(s))\|_{x} d s \leq \varepsilon+C_{2 R}\left(t-t_{0}\right)
\end{aligned}
$$

where we have used the contraction property of $S$ 
Now we return to the proof of Lemma 6.1. If we set

$$
t_{1}=t_{0}+\min \left\{\delta\left(x_{0}, \varepsilon\right), \frac{R-\varepsilon}{C_{2 R}}\right\}
$$

the assertion follws immediately.

Lemma 6.2. Under the assumptions of Proposition 6.1, for $t \in\left[t_{0}, t_{1}\right]$ the estimate

$$
\|F(\phi)(t)-F(\psi)(t)\|_{X} \leq D_{2 R} \int_{t_{0}}^{t}\|\phi(s)-\psi(s)\|_{X} d s
$$

holds for every $\phi, \psi \in B\left(x_{0}, R\right)$ where $D_{2 R}$ is the constant from Lemma 4.2 .

Proof. From Lemma 4.2 we obtain

$$
\begin{aligned}
\|F(\phi)(t)-F(\psi)(t)\|_{X} & \leq \int_{t_{0}}^{t}\|S(t-s)(G(\phi)(s)-G(\psi)(s))\|_{X} d s \\
& \leq \int_{t_{0}}^{t}\|G(\phi)(s)-G(\psi)(s)\|_{X} d s \\
& \leq D_{2 R} \int_{t_{0}}^{t}\|\phi(s)-\psi(s)\|_{X} d s
\end{aligned}
$$

for every $\phi, \psi \in B\left(x_{0}, R\right)$

Finally, we can prove our main theorem.

Theorem 6.1. Let the parameters satisfy the assumptions

$$
\gamma_{1} \geq 0, \quad \gamma_{2} \geq 0, \quad \gamma_{3} \geq 0, \quad \gamma_{4} \leq 0, \quad \gamma_{5} \leq 0 .
$$

Then for every $T>0$ the Cauchy problem (2.5) - (2.6) admits a unique strong solution $x \in C([0, T], X)$.

Proof. Let $T>0$ be fixed. For $x \in X$ the mapping $t \mapsto S(t) x$ is uniformly continuous on $[0, T]$. Therefore, given $\varepsilon>0$, there exists a $\delta\left(x_{0}, \varepsilon\right)>0$ not depending on $t$ such that

$$
\left\|S\left(t_{0}\right) x_{0}-S\left(t_{1}\right) x_{0}\right\|_{x}<\varepsilon \quad \text { if }\left|t_{0}-t_{1}\right|<\delta\left(x_{0}, \varepsilon\right), t_{0}, t_{1} \in[0, T] .
$$

Now choose $R>0$ such that $\left\|x_{0}\right\|_{X} \leq R$ and $\left|\gamma_{i}\right| \leq R(i=2, \ldots, 5)$, and fix $\varepsilon>0$ with $\varepsilon<R$. From (6.4) we see that assumption (i) of Theorem 5.1 is satisfied for $t \in\left[0, t_{1}\right]$, where $t_{1}=\min \left\{\delta\left(x_{0}, \varepsilon\right), \frac{R-\varepsilon}{C_{2 R}}\right\}$.

Assumption (ii) of Theorem 5.1 follows from Lemma 6.2 (with $K_{R}=D_{2 R}$ ). Therefore we obtain a solution $x$ of the integral equation (6.2) in the interval $\left[0, t_{1}\right]$ which is unique by Theorem 5.1. Now if $t_{1}<T$, we set $x_{1}=x\left(t_{1}\right)$ and repeat the argument 
above for the interval $t_{1} \leq t \leq t_{2}=t_{1}+\min \left\{\delta\left(x_{1}, \varepsilon\right), \frac{R-\varepsilon}{C_{2 R}}\right\}$. To show that this procedure leads to a solution in the whole interval $[0, T]$, it is sufficient to prove that any solution of problem (2.5)-(2.6) is a priori bounded for every $t \in[0, T]$. To show this, we take the scalar product of $(2.5)$ with a solution $x(t)$ in $X$ and obtain

$$
\frac{1}{2} \frac{d}{d t}\|x\|_{X}^{2}-\left\langle A_{0} x, x\right\rangle_{X}-\langle G(x), x\rangle_{X}=0
$$

Since $\left\langle A_{0} x, x\right\rangle_{X} \leq 0$, we have

$$
\frac{1}{2} \frac{d}{d t}\|x\|_{X}^{2}-\left(\int_{0}^{1} \gamma_{2} \partial_{\xi}^{3} u \partial_{\xi} v+\gamma_{3}\left\|\partial_{\xi} u\right\|_{0}^{2} \partial_{\xi}^{3} u \partial_{\xi} v+\gamma_{4}\left(\partial_{\xi} v\right)^{2}+\gamma_{5} \partial_{\xi}\left(v^{3}\right) \partial_{\xi} v d x\right) \leq 0
$$

and from $\gamma_{5} \partial_{\xi}\left(v^{3}\right) \partial_{\xi} v=3 \gamma_{5}\left(\partial_{\xi} v\right)^{2} v^{2} \leq 0$ we claim

$$
\frac{1}{2} \frac{d}{d t}\|x\|_{X}^{2}-\gamma_{2}\left\|\partial_{\xi}^{3} u\right\|_{0}\left\|\partial_{\xi} v\right\|_{0}-\left|\gamma_{4}\right|\left\|\partial_{\xi} v\right\|_{0}^{2}-\gamma_{3}\left\|\partial_{\xi} u\right\|_{0}^{2}\left\|\partial_{\xi}^{3} u\right\|_{0}\left\|\partial_{\xi} v\right\|_{0} \leq 0
$$

In the Appendix, Lemma $A .2$, we shall show that $\left\|\partial_{\xi} u\right\|_{0}$ is uniformly bounded for $t \geq 0$ if $\gamma_{3}>0$. As a consequence, we obtain the estimate $\frac{d}{d t}\|x\|_{X}^{2} \leq C\|x\|_{X}^{2}$ with a constant $C$ depending only on $\gamma_{2}, \gamma_{3}, \gamma_{4}$ and $\left\|\partial_{\xi} u\right\|_{0}$. Now the uniform Gronwall lemma (cf. [12: p. 89]) shows that $\|x\|_{X}^{2}$ is bounded for all $t \in[0, T]$ and the solution of problem (2.5) - (2.6) exists for all time.

To show the differentiability a.e. in $(0, T)$ of the solution, we note that $G$ satisfies a Lipschitz condition in an appropriate $R$-ball in $X$ with Lipschitz constant $D_{2 R}$ (see Lemma 4.2). We get

$$
\begin{aligned}
x(t+h)-x(t)= & S(t+h) x_{0}-S(t) x_{0}+\int_{0}^{t+h} S(t+h-s) G(x(s)) d s \\
& -\int_{0}^{t} S(t-s) G(x(s)) d s \\
= & S(t+h) x_{0}-S(t) x_{0}+\int_{0}^{h} S(t+h-s) G(x(s)) d s \\
& +\int_{0}^{t} S(t-s)[G(x(s+h))-G(x(s))] d s .
\end{aligned}
$$

Since $S(\cdot) x_{0}$ is differentiable for $x_{0} \in D\left(A_{0}\right)$, the mapping $s \mapsto S(t+h-s) G(x(s))$ is continuous, $S$ is a contraction and $G$ satisfies a Lipschitz condition, we obtain with suitable constants $c$ and $C$

$$
\begin{aligned}
\|x(t+h)-x(t)\|_{x} & \leq h c\left\|A_{0} x_{0}\right\|_{x}+h C_{2 R}+D_{2 R} \int_{0}^{t}\|x(s+h)-x(s)\|_{x} d s \\
& =h C+D_{2 R} \int_{0}^{t}\|x(s+h)-x(s)\|_{x} d s .
\end{aligned}
$$


From the Gronwall Lemma it follows that

$$
\|x(t+h)-x(t)\| x \leq C \exp \left(T D_{2 R}\right) h
$$

i.e. $x$ is Lipschitz continuous. Consequently, $G(x(\cdot))$ is Lipschitz continuous in $(0, T)$. Then the Cauchy problem $\frac{d y}{d t}(t)=A_{0} y(t)+G(x(t)), y(0)=x_{0}$ has a unique solution $y \in C([0, T], X)$, differentiable almost everywhere, with $\frac{d y}{d t} \in L^{1}((0, T), X)$ and $y(t) \in$ $D\left(A_{0}\right)$ a.c., satisfying

$$
y(t)=S(t) x_{0}+\int_{0}^{t} S(t-s) G(x(s)) d s=x(t)
$$

(see [9: Corollary 4.2.11]). This proves the theorem

\section{Appendix}

We assume that the parameters satisfy (6.6) and we use the notation $\dot{u}=\partial_{t} u$ and $u^{\prime}=\partial_{\xi} u$.

Lemma A.1. If $x(t)=(u(t), v(t))$ is a solution of equation (2.5), then $\|v\|_{0} \cdot$ is uniformly bounded for all $t \geq 0$.

Proof. We define the functional

$$
L_{1}(t)=\frac{1}{2}\left(\|v\|_{0}^{2}+\gamma_{2}\left\|u^{\prime}\right\|_{0}^{2}+\left\|u^{\prime \prime}\right\|_{0}^{2}+\frac{\gamma_{3}}{2}\left\|u^{\prime}\right\|_{0}^{4}\right)
$$

Taking the time-derivative of this functional leads to.

$$
\frac{d}{d t} L_{1}(t)=(v, \dot{v})_{0}+\gamma_{2}\left(u^{\prime}, v^{\prime}\right)_{0}+\left(u^{\prime \prime}, v^{\prime \prime}\right)_{0}+\gamma_{3}\left\|u^{\prime}\right\|_{0}^{2}\left(u^{\prime}, v^{\prime}\right)_{0}
$$

Since $(u, v)$ is a solution, we have

$$
\begin{aligned}
(v, \dot{v})_{0}= & -\left(v^{\prime \prime}, u^{\prime \prime}\right)_{0}-\gamma_{1}\left(\dot{v}_{1}^{\prime \prime}, v^{\prime \prime}\right)_{0}+\dot{\gamma}_{2}\left(v, u^{\prime \prime}\right)_{0} \\
& +\gamma_{3}\left\|u^{\prime}\right\|_{0}^{2}\left(v, u^{\prime \prime}\right)_{0}+\gamma_{4}(v, v)_{0}+\gamma_{5}\left(v, v^{3}\right)_{0} .
\end{aligned}
$$

Inserting (A.2) into (A.1), we obtain

$$
\frac{d}{d t} L_{1}(t)=-\gamma_{1}\left\|v^{\prime \prime}\right\|_{0}^{2}+\gamma_{4}\|v\|_{0}^{2}+\gamma_{5}\left\|v^{2}\right\|_{0}^{2} \leq \gamma_{4}\|v\|_{0}^{2}+\gamma_{5}\left\|v^{2}\right\|_{0}^{2}
$$

On the other hand, we have

$$
L_{1}(t) \geq \frac{1}{2}\|v\|_{0}^{2}
$$

Since $L_{1}(t)$ is decreasing by (A.3), we infer from (A.4) that $\|v\|_{0}$ is bounded for all $t$ 
Lemma A.2. If $x(t)=(u(t), v(t))$ is a solution of equation (2.5), then $\left\|u^{\prime}\right\|_{0}$ is uniformly bounded for all $t \geq 0$.

Proof: We introduce the functional

$$
L_{2}(t)=\frac{1}{2}\left(\|v\|_{0}^{2}+\|u\|_{0}^{2}+\left(1+\gamma_{1}\right)\left\|u^{\prime \prime}\right\|_{0}^{2}+\frac{\gamma_{3}}{2}\left\|u^{\prime}\right\|_{0}^{4}+(u, v)_{0}\right) .
$$

Then we get

$$
\begin{aligned}
\frac{d}{d t} L_{2}(t)= & (v, \dot{v})_{0}+(u, v)_{0}+\left(1+\gamma_{1}\right)\left(u^{\prime \prime}, v^{\prime \prime}\right)_{0} \\
& +\gamma_{3}\left\|u^{\prime}\right\|_{0}^{2}\left(u^{\prime}, v^{\prime}\right)_{0}+\|v\|_{0}^{2}+(u, \dot{v})_{0}
\end{aligned}
$$

For a solution $(u, v)$ we have

$$
\begin{aligned}
(u, \dot{v})_{0}= & -\left(u^{\prime \prime}, u^{\prime \prime}\right)_{0}-\gamma_{1}\left(u^{\prime \prime}, v^{\prime \prime}\right)_{0}+\gamma_{2}\left(u, u^{\prime \prime}\right)_{0} \\
& +\gamma_{3}\left\|u^{\prime}\right\|_{0}^{2}\left(u, u^{\prime \prime}\right)_{0}+\gamma_{4}(u, v)_{0}+\gamma_{5}\left(u, v^{3}\right)_{0} .
\end{aligned}
$$

Inserting (A.6) and (A.2) into (A.5), we obtain

$$
\begin{aligned}
\frac{d}{d t} L_{2}(t)= & \left(1+\gamma_{4}\right)(u, v)_{0}+\left(1+\gamma_{4}\right)\|v\|_{0}^{2}-\gamma_{1}\left\|v^{\prime \prime}\right\|_{0}^{2}+\gamma_{2}\left(v^{\prime \prime}, u\right)_{0}+\gamma_{5}\left\|v^{2}\right\|_{0}^{2} \\
& -\left\|u^{\prime \prime}\right\|_{0}^{2}-\gamma_{2}\left\|u^{\prime}\right\|_{0}^{2}-\gamma_{3}\left\|u^{\prime}\right\|_{0}^{4}+\gamma_{5}\left(u, v^{3}\right)_{0} .
\end{aligned}
$$

Using the Young inequality $a b \leq \frac{1}{2}\left(\varepsilon a^{2}+\frac{1}{\varepsilon} b^{2}\right)$, we get

$$
\left|\gamma_{2}\left(v^{\prime \prime}, \dot{u}\right)_{0}\right| \leq \frac{\gamma_{2}}{2}\left(\varepsilon\left\|v^{\prime \prime}\right\|_{0}^{2}+\frac{1}{\varepsilon}\|u\|_{0}^{2}\right)
$$

and for $\varepsilon$ small enough, such that $\frac{\varepsilon \gamma_{2}}{2} \leq \gamma_{1}$, we obtain.

$$
\gamma_{2}\left(v^{\prime \prime}, u\right)_{0}-\gamma_{1}\left\|v^{\prime \prime}\right\|_{0}^{2} \leq \frac{\gamma_{2}}{2 \varepsilon}\|u\|_{0}^{2} .
$$

Again by the Young inequality (with $\varepsilon=1$ ) and Lemma A.1 we get

$$
\left.\begin{array}{rl}
\left|\left(1+\gamma_{4}\right)(u, v)_{0}\right| & \leq\left|\left(1+\gamma_{4}\right)\right| \frac{1}{2}\left(\|u\|_{0}^{2}+\|v\|_{0}^{2}\right) \leq c_{1}\|u\|_{0}^{2}+c_{2} \\
\left|\gamma_{5}\left(u, v^{3}\right)_{0}\right| & \leq \frac{\left|\gamma_{5}\right|}{2}\left(\|u\|_{0}^{2}+\left\|v^{3}\right\|_{0}^{2}\right) \leq c_{3}\|u\|_{0}^{2}+c_{4} \quad . .
\end{array}\right\}
$$

with suitable constants $c_{i}$. Since $\|v\|_{0}^{2}$ is bounded by Lemma A.1, this implies

$$
\frac{d}{d t} L_{2}(t) \leq C_{1}+C_{2}\|u\|_{0}^{2}-\gamma_{2}\left\|u^{\prime}\right\|_{0}^{2}-\gamma_{3}\left\|u^{\prime}\right\|_{0}^{4} \leq C_{1}+C_{3}\left\|u^{\prime}\right\|_{0}^{2}-\gamma_{3}\left\|u^{\prime}\right\|_{0}^{4}
$$

where $C_{i}$ are appropriate positive constants. On the other hand,

$$
\begin{aligned}
L_{2}(t) & \geq \frac{1}{2}\left(\|v\|_{0}^{2}+\|u\|_{0}^{2}+\left(1+\gamma_{1}\right)\left\|u^{\prime \prime}\right\|_{0}^{2}-\frac{1}{2}\|u\|_{0}^{2}-\frac{1}{2}\|v\|_{0}^{2}\right) \\
& \geq \frac{1}{2}\left(\left(1-\frac{1}{2}\right)\|v\|_{0}^{2}+\left(1-\frac{1}{2}\right)\|u\|_{0}^{2}+\left(1+\gamma_{1}\right)\left\|u^{\prime \prime}\right\|_{0}^{2}\right) \\
& \geq C_{4}\left(\|u\|_{0}^{2}+\left\|u^{\prime \prime}\right\|_{0}^{2}\right) .
\end{aligned}
$$

We conclude that $\left\|u^{\prime}\right\|_{0}^{2} \leq C_{5} L_{2}(t)$ and for $\left\|u^{\prime}\right\|_{0}$ large, (A.7) implies $\frac{d L_{2}}{d t} \leq 0$. This shows that $\left\|u^{\prime}\right\|_{0}$ must be bounded for all $t \geq 0$

Acknowledgement. W. Herfort would like to thank the Außenamt of the Freie - Universität Berlin for the invitation to visit the Mathematical Institute during the winter and summer term in 1991/92. 


\section{References}

[1] Adams, R. A.: Sobolev Spaces. New York - San Francisco - London: Academic Press 1975.

[2] Blevins, R. D.: Flow-Induced Vibrations. New York: van Nostrand Reinhold Comp. 1977.

[3] Bonander, C.: Eine spezielle nichtlineare Balkengleichung. Existenz und Eindeutigkeit der Lösung. Diplomarbeit. Berlin: Freie Universität 1955.

[4] Edward, R. E.: Functional Analysis. New York etc.: Holt, Rinehart \& Winston 1965.

[5] Foias, C., Gussi, G. and V. Poenaru: Sur les solutions généralisées de certaines équations linéaires et quasi linéaires dans l'espace de Banach. Rev. Roum. Math. Pures Appl. 3 (1958), $283-304$

[6] Holmes, P. and J. Marsden: Bifurcation to divergence and flutter in flow induced oscillations. Automatica 14 (1978), 367 - 384.

[7] Herfort, W. and H. Troger: Robust modelling of flow induced oscillations of bluff bodies. Math. Modelling 8 (1987), 251 - 255.

[8] Nayfeh, A. H. and D. T. Mook: Nonlinear Oscillations. New York etc.: J. Wiley \& Sons 1979.

[9] Pazy, A.: Semigroups of Linear Operators and Applications to Partial Differential Equations. New York etc.: Springer-Verlag 1983.

[10] Parkinson, G. V. and N. P. H. Brooks: On the aeroelastic instability of bluff cylinders. J. Appl. Mech. 28 (1961), $252-258$.

[11] Showalter, R. E.: Hilbert Space Methods for Partial Differential Equations. London-San Francisco - Melbourne: Pitman 1977.

[12] Temam, R.: Infinite Dimensional Dynamical Systems in Mechanics and Physics. New York etc.: Springer-Verlag 1988. 